\title{
Pattern Formation in a Bacterial Colony Model
}

\author{
Xinze Lian, ${ }^{1,2}$ Guichen Lu, ${ }^{3}$ and Hailing Wang ${ }^{4}$ \\ ${ }^{1}$ School of Mathematics, Wenzhou University, Wenzhou 325000, China \\ ${ }^{2}$ Chengdu Institute of Computer Application, Chinese Academy of Sciences, Chengdu 610041, China \\ ${ }^{3}$ School of Mathematics and Statistics, Chongqing University of Technology, Chongqing 400054, China \\ ${ }^{4}$ Department of Mathematics, Hubei Minzu University, Enshi, Hubei 445000, China
}

Correspondence should be addressed to Xinze Lian; xinzelian@163.com and Guichen Lu; bromn006@gmail.com

Received 2 January 2014; Accepted 8 February 2014; Published 23 March 2014

Academic Editor: Kaifa Wang

Copyright (C) 2014 Xinze Lian et al. This is an open access article distributed under the Creative Commons Attribution License, which permits unrestricted use, distribution, and reproduction in any medium, provided the original work is properly cited.

We investigate the spatiotemporal dynamics of a bacterial colony model. Based on the stability analysis, we derive the conditions for Hopf and Turing bifurcations. Furthermore, we present novel numerical evidence of time evolution of patterns controlled by parameters in the model and find that the model dynamics exhibit a diffusion controlled formation growth to spots, holes and stripes pattern replication, which show that the bacterial colony model is useful in revealing the spatial predation dynamics in the real world.

\section{Introduction}

Spatial patterns which are formed by some kinds of bacterial colonies present an interesting structure during their growth conditions. In particular, colonies of bacterium bacillus subtilis can present a rich variety of structures [1-13]. The nature of the pattern exhibited depends on the particular bacterial species used and the environmental conditions imposed. Ohgiwari et al. [11] have shown that for a nutrient-poor solid agar, the bacterium colonies exhibit fractal morphogenesis similar to diffusion-limited aggregation (DLA). For softer agar medium, the colonies tend to show a dense-branching morphology (DBM) [7]. If both the nutrient concentration and the agar's softness further increase, simple circular colonies grow almost homogeneously in space [14].

There are many mathematical models for explaining each characteristic colony pattern. Kawasaki et al. [7] have developed a reaction-diffusion model and have shown the patterns by using the computer simulations. Since in Kawasaki et al.s model, all the nutrients must be consumed; L. Braverman and E. Braverman [4] have introduced a model of prey-predator type with Holling-II functional response under the situation of a renewable nutrient. In the present paper, motivated by the work of L. Braverman and E. Braverman, we consider the model with the consumption term of nutrient in a Holling III functional response.

Let us denote by $u(t, x, y)$ and $v(t, x, y)$ the nutrient concentration and the density of the bacterial cells at point $(x, y)$, respectively. We consider the following system:

$$
\begin{aligned}
& \frac{\partial u}{\partial t}=D_{u} \nabla^{2} u-\frac{\kappa u^{2} v}{v^{2}+\gamma_{0}^{2} u^{2}}+r u\left(1-\frac{u}{M}\right), \\
& \frac{\partial v}{\partial t}=\nabla \cdot\left(D_{v} \nabla v\right)+\theta \frac{\kappa u^{2} v}{v^{2}+\gamma_{0}^{2} u^{2}}-\gamma v,
\end{aligned}
$$

where $r$ is the intrinsic nutrient growth rate, $M$ is the carrying capacity of the environment for the nutrient (prey), $\gamma$ is the bacteria (predator) mortality rate, $\kappa, \theta$, and $\gamma_{0}$ are parameters of the Holling Type III functional response, and $D_{v}$ is the nutrient diffusion coefficient. Following [4, 7], we assumed that the diffusion coefficient is proportional to both nutrient and bacteria densities

$$
D_{v}=\sigma u v .
$$

Here we try to model the situation of a renewable nutrient. Then the system involves two reaction-diffusion equations of a predator-prey type with a Holling Type III 
functional response. Diffusive predator-prey systems were extensively studied; we mention here the recent papers [1519], the monograph [20], and the references therein. In the present paper, it is to investigate the spatial pattern formation of system (1) which means the convergence of solutions to some stable spatially-in-homogeneous pattern as time tends to infinity. And in natural science, the pattern formation can reveal the evolution process of the species; it is, perhaps, the most challenging in modern ecology, biology, chemistry, and many other fields of science [21-38]. Thus, our basic concern is to find, if any, a spatially inhomogeneous equilibrium and periodic solutions that are stable in a certain sense. From the pioneer work by Turing [12], it is widely known that a reaction-diffusion system exhibits Turing instability if the homogenous steady state is stable to small perturbations in the absence of diffusion but unstable to small spatial perturbations when diffusion is present which implies the existence of spatially in-homogenous solutions. From the Hopf bifurcation analysis and the phrase transition theory developed by Ma and Wang [39-42], it is shown that the periodic solutions exist [43].

The paper is organized as follows. In Section 2, we give the analysis of the model and mathematical setup. In Section 3, we analyze the spatial model, we derive the conditions of the Turing bifurcation and Hopf bifurcation, and we give the existence of periodic solution. We give some computer simulations to illustrate the emergence of pattern formation in Section 4. Finally, some conclusions are given.

\section{Modeling Analysis and Mathematical Setup}

To obtain the dimensionless form of the system (1), we introduce the following:

$$
\begin{gathered}
u=M u^{\prime}, \quad v=M \gamma_{0} v^{\prime}, \quad t=\frac{1}{r} t^{\prime}, \quad x=\left(\frac{1}{r}\right)^{1 / 2} x^{\prime}, \\
y=\left(\frac{1}{r}\right)^{1 / 2} y^{\prime}, \quad \gamma=\frac{1}{r} \gamma^{\prime}, \quad \sigma=\frac{1}{\gamma_{0} M^{2}} \sigma^{\prime} .
\end{gathered}
$$

Omitting the primes, we obtain the following nondimensional form of (1):

$$
\begin{aligned}
& \frac{\partial u}{\partial t}=D_{u} \nabla^{2} u-\alpha \frac{u^{2} v}{v^{2}+u^{2}}+u(1-u), \\
& \frac{\partial v}{\partial t}=\sigma \nabla \cdot(u v \nabla v)+\beta \frac{u^{2} v}{v^{2}+u^{2}}-\gamma v,
\end{aligned}
$$

with $\alpha=\kappa / r \gamma_{0}, \beta=\theta \kappa / r \gamma_{0}$.

Model (4) is to be analyzed under the following nonzero initial conditions:

$$
\begin{aligned}
& u(t, x, y)>0, \quad v(t, x, y)>0, \\
& (x, y) \in \Omega=\left(0, L_{x}\right) \times\left(0, L_{y}\right)
\end{aligned}
$$

and Neumann boundary conditions:

$$
\left.\frac{\partial u}{\partial \nu}\right|_{\partial \Omega}=\left.\frac{\partial v}{\partial \nu}\right|_{\partial \Omega}=0 .
$$

In the above, $L_{x}$ and $L_{y}$ denote the size of the system in square domain and $v$ is the outward unit normal vector of the boundary $\partial \Omega$. The main reason for choosing such boundary conditions is that we are interested in the self-organization of the pattern and the Neumann conditions imply no external input [22].

It is known that only nonnegative solutions of (4) have biological significance. System (4) has two spatially homogeneous stationary solutions:

(1) the bacteria-free equilibrium $U_{0}=(1,0)$ which implies that the nutrient is at the carrying capacity level;

(2) coexistence equilibrium $U^{*}=\left(u^{*}, v^{*}\right)$ which represents a uniform distribution of bacteria, where

$$
u^{*}=\frac{\beta-S \alpha}{\beta}, \quad v^{*}=\frac{S(\beta-S \alpha)}{\beta \gamma},
$$

and $S=\sqrt{\gamma(\beta-\gamma)}$ with $\beta>\gamma$ and $\beta^{2}-\alpha^{2} \gamma \beta+\alpha^{2} \gamma^{2}>0$.

To consider the pattern formation of $(4)$ from $\left(u^{*}, v^{*}\right)$ we make the translation

$$
u \longrightarrow u_{1}+u^{*}, \quad v \longrightarrow u_{2}+v^{*} .
$$

Then, (4) are rewritten as

$$
\begin{gathered}
\frac{\partial u_{1}}{\partial t}=D_{u} \nabla^{2} u_{1}+a_{11} u_{1}+a_{12} u_{2}+G_{1}\left(u_{1}, u_{2}\right), \\
\frac{\partial u_{2}}{\partial t}=\mu \nabla^{2} u_{2}+a_{21} u_{1}+a_{22} u_{2}+g\left(u_{1}, u_{2}\right)+G_{2}\left(u_{1}, u_{2}\right),
\end{gathered}
$$

where

$$
\begin{array}{ll}
a_{11}=\frac{-\beta^{2}+2 S \alpha \gamma}{\beta^{2}}, & a_{12}=-\frac{(2 \gamma-\beta) \gamma \alpha}{\beta^{2}}, \\
a_{21}=-2 \frac{(\gamma-\beta) S \alpha}{\alpha \beta}, & a_{22}=2 \frac{\gamma(\gamma-\beta)}{\beta},
\end{array}
$$

and $\mu=u^{*} v^{*} \sigma, g\left(u_{1}, u_{2}\right)=\sigma\left[\nabla \cdot\left(u_{1} u_{2} \nabla u_{2}\right)+v^{*} \nabla \cdot\left(u_{1} \nabla u_{2}\right)+\right.$ $\left.u^{*} \nabla \cdot\left(u_{2} \nabla u_{2}\right)\right], G_{1}\left(u_{1}, u_{2}\right)$, and $G_{2}\left(u_{1}, u_{2}\right)$ are terms of high order.

Define two Hilbert spaces

$$
\begin{gathered}
X=H^{2}(\Omega), \\
X_{1}=\left\{u \in H^{2}(\Omega, R) \mid \frac{\partial u}{\partial \nu} \text { on } \partial \Omega\right\} .
\end{gathered}
$$

Then $X_{1} \rightarrow X$ is dense and compact inclusion.

$$
L_{\lambda}:=-B_{\lambda}+A,
$$

where

$$
\begin{aligned}
-B_{\lambda} u & =\left(D_{u} \Delta u_{1}, \mu \Delta u_{2}\right)^{T}, \\
A u & =\left(\begin{array}{ll}
a_{11} & a_{12} \\
a_{21} & a_{22}
\end{array}\right)\left(\begin{array}{l}
u_{1} \\
u_{2}
\end{array}\right)
\end{aligned}
$$

for $u=\left(u_{1}, u_{2}\right)^{T} \in X_{1}$. 
Furthermore, denote that

$$
\begin{aligned}
& G(u, \lambda) \\
& =\left(G_{1}^{2}(u, \lambda)+G_{1}^{3}(u, \lambda)+g_{1}\left(u_{1}, u_{2}\right)\right. \\
& \left.\quad G_{2}^{2}(u, \lambda)+G_{2}^{3}(u, \lambda)+g\left(u_{1}, u_{2}\right)+g_{2}\left(u_{1}, u_{2}\right)\right)^{T}
\end{aligned}
$$

with

$$
\begin{gathered}
\left(\begin{array}{c}
G_{1}^{2}(u, \lambda) \\
G_{2}^{2}(u, \lambda)
\end{array}\right)=\left(\begin{array}{c}
a_{20} u_{1}^{2}+a_{11} u_{1} u_{2}+a_{02} u_{2}^{2} \\
b_{20} u_{1}^{2}+b_{11} u_{1} u_{2}+b_{02} u_{2}^{2}
\end{array}\right), \\
\left(\begin{array}{l}
G_{1}^{3}(u, \lambda) \\
G_{2}^{3}(u, \lambda)
\end{array}\right)=\left(\begin{array}{c}
a_{30} u_{1}^{3}+a_{21} u_{1}^{2} u_{2}+a_{12} u_{1} u_{2}^{2}+a_{03} u_{2}^{3} \\
b_{30} u_{1}^{3}+b_{21} u_{1}^{2} u_{2}+b_{12} u_{1} u_{2}^{2}+b_{03} u_{2}^{3}
\end{array}\right),
\end{gathered}
$$

where

$$
\begin{aligned}
& a_{20}=-\frac{-\beta^{3}-4 S \alpha \gamma^{2}+5 S \alpha \beta \gamma}{\beta^{2}(-\beta+S \alpha)}, \\
& a_{11}=-2 \frac{\alpha \gamma(-\gamma+\beta)(\beta-4 \gamma)}{\beta^{2}(-\beta+S \alpha)}, \\
& a_{02}=\frac{(\beta-4 \gamma) S \alpha \gamma}{\beta^{2}(-\beta+S \alpha)}, \\
& a_{30}=4 \frac{(\beta-2 \gamma)(\beta-\gamma) S \alpha \gamma}{\beta^{2}(-\beta+S \alpha)^{2}}, \\
& a_{21}=\frac{\alpha \gamma(\beta-\gamma)\left(24 \gamma^{2}-16 \gamma \beta+\beta^{2}\right)}{\beta^{2}(-\beta+S \alpha)^{2}}, \\
& a_{12}=-2 \frac{\left(-10 \gamma \beta+\beta^{2}+12 \gamma^{2}\right) S \alpha \gamma}{\beta^{2}(-\beta+S \alpha)^{2}} \text {, } \\
& a_{03}=\frac{\alpha \gamma^{2}\left(-8 \gamma \beta+\beta^{2}+8 \gamma^{2}\right)}{\beta^{2}(-\beta+S \alpha)^{2}}, \\
& b_{20}=-\frac{S \alpha(\beta-\gamma)(\beta-4 \gamma)}{\alpha \beta(-\beta+S \alpha)} \text {, } \\
& b_{11}=\frac{2 \gamma(\beta-\gamma)(\beta-4 \gamma)}{\beta(-\beta+S \alpha)} \text {, } \\
& b_{02}=-\frac{(\beta-4 \gamma) S \gamma}{\beta(-\beta+S \alpha)}, \\
& b_{30}=-\frac{4(\beta-2 \gamma)(\beta-\gamma) S \gamma}{\beta(-\beta+S \alpha)^{2}}, \\
& b_{21}=-\frac{\gamma(\beta-\gamma)\left(24 \gamma^{2}-16 \gamma \beta+\beta^{2}\right)}{\beta(-\beta+S \alpha)^{2}} \text {, }
\end{aligned}
$$

$$
\begin{aligned}
& b_{12}=\frac{2\left(-10 \gamma \beta+\beta^{2}+12 \gamma^{2}\right) S \gamma}{\beta(-\beta+S \alpha)^{2}}, \\
& b_{03}=-\frac{\gamma^{2}\left(-8 \gamma \beta+\beta^{2}+8 \gamma^{2}\right)}{\beta(-\beta+S \alpha)^{2}} .
\end{aligned}
$$

Here $g_{1}\left(u_{1}, u_{2}\right)$ and $g_{2}\left(u_{1}, u_{2}\right)$ are terms of high order.

Then $G(\cdot, \lambda): X_{1} \rightarrow X$ are a family of parameterized $\mathbb{C}^{\infty}$ bounded operators continuously depending on the parameter $\lambda$ such that $G(u, \lambda)=o(\|u\|)$.

Then (9) can be written in the following operator form:

$$
\frac{d u}{d t}=F(u)=L_{\lambda} u+G(u, \lambda) .
$$

\section{Bifurcation Analysis}

Unless otherwise specified, in this section, we require that $U^{*}=\left(u^{*}, v^{*}\right)$ always exist; that is, $\beta>\gamma$ and $\beta^{2}-\alpha^{2} \gamma \beta+$ $\alpha^{2} \gamma^{2}>0$.

Consider the following eigenvalue problem of system (9):

$$
L_{\lambda} \varphi=\lambda \varphi, \quad \varphi \in H_{1}
$$

with the Neumann boundary condition (6).

Let $\rho_{k}$ and $e_{k}$ be the $k$ th eigenvalue and eigenvector of the Laplacian $\nabla^{2}$ with Neumann boundary condition and

$$
\begin{aligned}
-\nabla^{2} e_{k} & =\rho_{k} e_{k}, \\
\left.\frac{\partial e_{k}}{\partial \nu}\right|_{\partial \Omega} & =0
\end{aligned}
$$

with $\rho_{0}=0, e_{0}=(1,1)^{T}$.

Denote by $M_{k}$ the matrix given by

$$
M_{k}=\left(\begin{array}{cc}
a_{11}-D_{u} \rho_{k} & a_{12} \\
a_{21} & a_{22}-\mu \rho_{k}
\end{array}\right), \quad k=0,1,2, \ldots
$$

Thus, all eigenvalues $\lambda=\beta_{k}^{ \pm}$of (18) satisfy

$$
M_{k} \xi_{k}^{ \pm}=\beta_{k} \xi_{k}^{ \pm}, \quad k=0,1,2, \ldots
$$

where $\xi_{k}^{ \pm} \in \mathbb{R}^{2}$ is the eigenvector of $M_{k}$ corresponding to $\beta_{k}^{ \pm}$ and $\beta_{k}^{ \pm}$is expressed as

$$
\beta_{k}^{ \pm}=\frac{1}{2}\left(\operatorname{tr}\left(M_{k}\right) \pm \sqrt{\operatorname{tr}\left(M_{k}\right)^{2}-4 \operatorname{det}\left(M_{k}\right)}\right)
$$

with

$$
\begin{aligned}
\operatorname{tr}\left(M_{k}\right) & =\left(D_{u} \rho_{k}-a_{11}\right)+\left(\mu \rho_{k}-a_{22}\right), \\
\operatorname{det}\left(M_{k}\right) & =\left(D_{u} \rho_{k}-a_{11}\right)\left(\mu \rho_{k}-a_{22}\right)-a_{12} a_{21} .
\end{aligned}
$$

Hence, the eigenvector $\phi_{k}^{ \pm}$of (18) corresponding to $\beta_{k}^{ \pm}$is

$$
\phi_{k}^{ \pm}=\xi_{k}^{ \pm} e_{k}
$$

where $e_{k}$ is as in (19). 
3.1. Hopf Bifurcation Analysis. It is clear that $\beta_{k}^{ \pm}(\alpha)= \pm i \sigma_{k}(\alpha)$ with $\sigma_{k} \neq 0$ if and only if

$$
\begin{gathered}
\operatorname{tr}\left(M_{k}\right)=\left(a_{11}-D_{u} \rho_{k}\right)+\left(a_{22}-\mu \rho_{k}\right)=0, \\
\operatorname{det}\left(M_{k}\right)=\left(D_{u} \rho_{k}-a_{11}\right)\left(\mu \rho_{k}-a_{22}\right)-a_{12} a_{21}>0 .
\end{gathered}
$$

Thus, we introduce one critical number

$$
\alpha_{0}=\frac{\beta\left(\beta-2 \gamma^{2}+2 \gamma \beta\right)}{2 S \gamma},
$$

where $\rho_{k}=\rho_{0}=0$ such that $\chi(\alpha)$ attains its minimum values. Consider

$$
\begin{aligned}
\chi(\alpha) & =\min _{\rho_{k}}\left\{\left(D_{u} \rho_{k}+a_{11}\right)\left(\mu \rho_{k}+a_{22}\right)-a_{12} a_{21}\right\} \\
& =a_{11} a_{22}-a_{12} a_{21} .
\end{aligned}
$$

Theorem 1. Let $\alpha_{0}$ be the number given in (26) such that (27) is satisfied. Then $\beta_{0}^{+}(\lambda)$ and $\beta_{0}^{-}(\lambda)$ are a pair of first complex eigenvalues of (18) near $\lambda=\alpha_{0}$, and

$$
\begin{gathered}
\operatorname{Re} \beta_{0}^{+}(\lambda)=\operatorname{Re} \beta_{0}^{-}(\lambda) \begin{cases}<0, & \lambda<\alpha_{0}, \\
=0, & \lambda=\alpha_{0}, \\
>0, & \lambda>\alpha_{0},\end{cases} \\
\quad \operatorname{Im} \beta_{0}^{ \pm}\left(\alpha_{0}\right) \neq 0, \\
\operatorname{Re} \beta_{k}^{ \pm}\left(\alpha_{0}\right)<0, \quad \forall k>0 .
\end{gathered}
$$

3.2. Periodic Solution from Hopf Bifurcation. By Theorem 1, problem (4) undergoes a dynamic transition to a periodic solution from $\alpha=\alpha_{0}$. To determine the types of transition we introduced a parameter as follows:

$$
b=\frac{F_{1}}{F_{2}},
$$

where

$$
\begin{aligned}
F_{1}= & \pi\left(\alpha^{2} \gamma S \beta-\alpha^{2} S \gamma^{2}-2 \beta \gamma^{2} \alpha+2 \alpha \gamma \beta^{2}+S \beta^{2}\right) \\
\times( & -8 \beta^{5} \gamma^{4} \alpha^{4}-20 \beta^{9} \gamma^{2}+512 \beta^{2} \gamma^{7} \alpha^{4} \\
& -416 \beta \gamma^{8} \alpha^{4}-66 \beta^{8} \gamma^{3} \omega^{2}+80 \beta^{7} \gamma^{4} \omega^{2} \\
& -32 \beta^{6} \omega^{2} \gamma^{5}+66 \beta^{8} \gamma^{3}+20 \beta^{9} \gamma^{2} \omega^{2} \\
& -2 \beta^{10} \gamma \omega^{2}+464 \beta^{5} \alpha^{2} \gamma^{5}-88 \beta^{6} \gamma^{5} \alpha^{2} \\
& +8 \beta^{7} \gamma^{4} \alpha^{2}-456 \beta^{4} \alpha^{2} \gamma^{6}+160 \beta^{3} \alpha^{2} \gamma^{7} \\
+ & 128 \alpha^{4} \gamma^{9}-296 \beta^{3} \gamma^{6} \alpha^{4}+80 \beta^{4} \gamma^{5} \alpha^{4} \\
& -202 \beta^{6} \alpha^{2} \gamma^{4}+2 \beta^{10} \gamma-16 \gamma^{5} \beta^{5} \omega \alpha
\end{aligned}
$$

$-2 \gamma \beta^{9} \omega \alpha+16 \gamma^{2} \beta^{8} \omega \alpha-42 \gamma^{3} \beta^{7} \omega \alpha$

$+44 \gamma^{4} \beta^{6} \omega \alpha-64 \alpha^{3} \beta^{2} \gamma^{6} \omega^{2} S+18 \alpha^{3} \beta^{5} \gamma^{3} \omega^{2} S$

$+120 \alpha^{3} \beta^{3} \gamma^{5} \omega^{2} S-76 \alpha^{3} \beta^{4} \gamma^{4} \omega^{2} S$

$-\alpha^{3} \beta^{6} \gamma^{2} \omega^{2} S+252 \alpha^{2} \beta^{5} \gamma^{4} \omega S-304 \alpha^{2} \beta^{4} \gamma^{5} \omega S$

$+128 \alpha^{2} \beta^{3} \gamma^{6} \omega S-86 \alpha^{2} \beta^{6} \gamma^{3} \omega S+10 \alpha^{2} \beta^{7} \gamma^{2} \omega S$

$-16 \alpha \beta^{7} \gamma^{2} \omega^{2} S+44 \alpha \beta^{6} \gamma^{3} \omega^{2} S-32 \alpha \beta^{5} \gamma^{4} \omega^{2} S$

$+\alpha \beta^{8} \gamma \omega^{2} S-2 \beta^{8} \alpha^{2} \gamma^{2}+2 \beta^{10} \omega S$

$-128 \alpha^{2} \beta^{2} \gamma^{9}-584 \alpha^{2} \beta^{4} \gamma^{7}+344 \alpha^{2} \beta^{5} \gamma^{6}$

$+448 \alpha^{2} \beta^{3} \gamma^{8}+36 \beta^{7} \gamma^{3} \alpha^{2}-80 \beta^{7} \gamma^{4}$

$+32 \beta^{6} \gamma^{5}-22 \beta^{9} \gamma \omega S+84 \beta^{8} \gamma^{2} \omega S$

$-128 \beta^{7} \gamma^{3} \omega S+64 \beta^{6} \gamma^{4} \omega S+340 \alpha^{3} \beta^{4} \gamma^{5} S$

$+4 \alpha^{3} \beta^{6} S \gamma^{3}-760 \alpha^{3} \beta^{3} \gamma^{6} S-72 \alpha^{3} \beta^{3} \gamma^{5} S$

$+2 \alpha^{3} \beta^{6} S \gamma^{2}-256 \alpha^{3} \beta \gamma^{8} S+56 \alpha^{3} \beta^{4} S \gamma^{4}$

$-18 \alpha^{3} \beta^{5} S \gamma^{3}-64 \alpha^{3} \beta^{5} S \gamma^{4}+32 \alpha^{3} \beta^{2} \gamma^{6} S$

$+736 \alpha^{3} \beta^{2} \gamma^{7} S-64 \alpha^{2} \beta^{3} \omega^{2} \gamma^{7}-120 \alpha^{2} \beta^{5} \omega^{2} \gamma^{5}$

$+152 \alpha^{2} \beta^{4} \omega^{2} \gamma^{6}+34 \alpha^{2} \beta^{6} \gamma^{4} \omega^{2}$

$-2 \alpha^{2} \beta^{7} \gamma^{3} \omega^{2}-4 \alpha \beta^{9} \omega \gamma^{2}+48 \alpha \beta^{8} \gamma^{3} \omega$

$-212 \alpha \beta^{7} \omega \gamma^{4}+424 \alpha \beta^{6} \gamma^{5} \omega$

$-384 \alpha \gamma^{6} \omega \beta^{5}+128 \alpha \gamma^{7} \omega \beta^{4}$

$-10 \alpha \beta^{8} \gamma^{2} \omega^{3}+46 \alpha \beta^{7} \gamma^{3} \omega^{3}$

$-68 \alpha \beta^{6} \gamma^{4} \omega^{3}+32 \alpha \beta^{5} \gamma^{5} \omega^{3}-28 \alpha \beta^{6} S \gamma^{3}$

$+14 \alpha \beta^{7} S \gamma^{2}-8 \alpha \beta^{8} \gamma^{2} S+80 \alpha \beta^{7} \gamma^{3} S$

$-264 \alpha \beta^{6} \gamma^{4} S+320 \alpha \beta^{5} \gamma^{5} S-128 \alpha \beta^{4} \gamma^{6} S$

$\left.+16 \alpha \beta^{5} S \gamma^{4}-2 \alpha \beta^{8} S \gamma\right)$,

$$
\begin{aligned}
F_{2}= & 4 \omega^{2}(-2 \gamma+\beta)^{2}\left(-\alpha^{2} \gamma^{2}-\beta^{2}+\alpha^{2} \gamma \beta\right)^{2} \\
& \times \beta^{3} \alpha^{2} \gamma^{2}(-\gamma+\beta) .
\end{aligned}
$$

Theorem 2. Let $b$ be the number given by (29), then the problem undergoes a transition to periodic solutions at $\lambda=\lambda_{0}$, and the following assertions hold true.

(1) When $b<0$, the transition is continuous and the system bifurcates to a periodic solution on $\alpha<\alpha_{0}$ which is an attractor. 
(2) When $b>0$, the transition is jump and the system bifurcates to a periodic solution on $\alpha>\alpha_{0}$ which is a repeller.

Proof. We will verify this theorem by using Theorem A.3 in [44]. The eigenvalues $\beta_{1}^{ \pm}$at $\lambda=\alpha_{0}$ in are given by $\beta_{1}^{+}=\bar{\beta}_{1}^{-}=$ $i \omega$. The eigenvectors $\xi$ and $\eta$ corresponding to $\beta_{1}^{ \pm}\left(\alpha_{0}\right)$ satisfy

$$
\begin{gathered}
A \xi=\omega \eta, \\
A \eta=-\omega \xi .
\end{gathered}
$$

It is easy to see that

$$
\begin{gathered}
\xi=\left(\xi_{1}, \xi_{2}\right)=\left(a_{11}, a_{12}\right), \\
\eta=\left(\eta_{1}, \eta_{2}\right)=(-\omega, 0) .
\end{gathered}
$$

The conjugate eigenvectors $\xi^{*}$ and $\eta^{*}$ satisfy

$$
\begin{gathered}
A \xi^{*}=\omega \eta^{*}, \\
A \eta^{*}=-\omega \xi^{*} .
\end{gathered}
$$

It is easy to check that

$$
\begin{gathered}
\xi^{*}=\left(\xi_{1}^{*}, \xi_{2}\right)=\left(a_{11}, a_{21}\right), \\
\eta^{*}=\left(\eta_{1}^{*}, \eta_{2}^{*}\right)=(-\omega, 0) .
\end{gathered}
$$

It is known that functions $\psi_{1}^{*}$ and $\psi_{2}^{*}$ are given by

$$
\begin{gathered}
\psi_{1}^{*}=\frac{1}{\left(\xi, \xi^{*}\right)}\left[\left(\xi, \xi^{*}\right) \xi^{*}+\left(\xi, \eta^{*}\right) \eta^{*}\right]=\left(0, a_{21}\right), \\
\psi_{2}^{*}=\frac{1}{\left(\eta, \eta^{*}\right)}\left[\left(\eta, \xi^{*}\right) \xi^{*}+\left(\eta, \eta^{*}\right) \eta^{*}\right] \\
=\left(\frac{a_{12} a_{21}}{\omega},-\frac{a_{11} a_{21}}{\omega}\right) .
\end{gathered}
$$

Because the first eigenvector space $E=\operatorname{span}\{\xi, \eta\}$ of (18) with (6) is invariant for the equations (4) with (6), the center manifold function $\Phi$ vanishes; that is,

$$
\Phi(x, y) \equiv 0 .
$$

Therefore, we derive from (32) to (35) that

$$
\begin{aligned}
\frac{G(x \xi+y \eta+\Phi), \psi_{1}^{*}}{\left(\xi, \psi_{1}^{*}\right)} & \\
= & \bar{a}_{20} x^{2}+\bar{a}_{11} x y+\bar{a}_{02} y^{2} \\
& +\bar{a}_{30} x^{3}+\bar{a}_{21} x^{2} y+\bar{a}_{12} x y^{2}+\bar{a}_{03} y^{3}, \\
\frac{G(x \xi+y \eta+\Phi), \psi_{2}^{*}}{\left(\eta, \psi_{2}^{*}\right)} & \bar{b}_{20} x^{2}+\bar{b}_{11} x y+\bar{b}_{02} y^{2}+\bar{b}_{30} x^{3} \\
& +\bar{b}_{21} x^{2} y+\bar{b}_{12} x y^{2}+\bar{b}_{03} y^{3},
\end{aligned}
$$

where

$$
\begin{aligned}
& \bar{a}_{20}=\frac{1}{a_{12}}\left(b_{11} a_{11} a_{12}+b_{02} a_{12}^{2}+b_{20} a_{11}^{2}\right), \\
& \bar{a}_{11}=\frac{\omega}{a_{12}}\left(2 b_{20} a_{11}+b_{11} a_{12}\right) \bar{a}_{02}=\frac{b_{20} \omega^{2}}{a_{12}}, \\
& \bar{a}_{30}=\frac{1}{a_{12}}\left(b_{21} a_{11}^{2} a_{12}+b_{30} a_{11}^{3}\right. \\
& \left.+b_{12} a_{11} a_{12}^{2}+b_{03} a_{12}^{3}\right), \\
& \bar{a}_{12}=\frac{\omega^{2}}{a_{12}}\left(b_{21} a_{12}+3 b_{30} a_{11}\right), \\
& \bar{a}_{21}=-\frac{\omega}{a_{12}}\left(3 b_{30} a_{11}^{2}+b_{12} a_{12}^{2}+2 b_{21} a_{11} a_{12}\right), \\
& \bar{a}_{03}=-\frac{b_{30} \omega^{3}}{a_{12}}, \\
& \bar{b}_{11}=-\frac{a_{11}}{a_{12}}\left(-2 a_{12} a_{20}-a_{12}^{2}+2 b_{20} a_{11}+b_{11} a_{12}\right) \text {, } \\
& \bar{b}_{02}=\frac{\omega}{a_{12}}\left(-a_{12} a_{20}+b_{20} a_{11}\right), \\
& \bar{b}_{20}=-\frac{1}{a_{12} \omega}\left(a_{11}^{2} a_{12}^{2}+a_{02} a_{12}^{3}+a_{12} a_{20} a_{11}^{2}\right. \\
& \left.-b_{11} a_{11}^{2} a_{12}-a_{11} b_{02} a_{12}^{2}-b_{20} a_{11}^{3}\right), \\
& \bar{b}_{30}=-\frac{1}{a_{12} \omega}\left(a_{11}^{2} a_{12}^{2} a_{21}+a_{12} a_{30} a_{11}^{3}+a_{12}^{4} a_{11}\right. \\
& +a_{03} a_{12}^{4}-b_{21} a_{11}^{3} a_{12}-b_{30} a_{11}^{4} \\
& \left.-b_{12} a_{11}^{2} a_{12}^{2}-a_{11} b_{03} a_{12}^{3}\right), \\
& \bar{b}_{21}=-\frac{1}{a_{12}}\left(-3 a_{12} a_{30} a_{11}^{2}-a_{12}^{4}\right. \\
& -2 a_{21} a_{11} a_{12}^{2}+3 b_{30} a_{11}^{3} \\
& \left.+b_{12} a_{11} a_{12}^{2}+2 b_{21} a_{11}^{2} a_{12}\right), \\
& \bar{b}_{12}=\frac{\omega}{a_{12}}\left(-a_{12}^{2} a_{21}-3 a_{12} a_{30} a_{11}\right. \\
& \left.+b_{21} a_{11} a_{12}+3 b_{30} a_{11}^{2}\right), \\
& \bar{b}_{03}=-\frac{\omega^{2}}{a_{12}}\left(-a_{12} a_{30}+b_{30} a_{11}\right) \text {. }
\end{aligned}
$$

From the focus values in $[39,40,43]$, we have that $b=\frac{3 \pi}{4}\left(\bar{a}_{30}+\bar{b}_{03}\right)+\frac{\pi}{4}\left(\bar{a}_{12}+\bar{b}_{21}\right)$ 


$$
\begin{aligned}
& +\frac{\pi}{2 \omega}\left(\bar{a}_{02} \bar{b}_{02}-\bar{a}_{20} \bar{b}_{20}\right) \\
& +\frac{\pi}{4 \omega}\left(\bar{a}_{11} \bar{a}_{20}+\bar{a}_{11} \bar{a}_{02}-\bar{b}_{11} \bar{b}_{20}-\bar{b}_{11} \bar{b}_{02}\right)
\end{aligned}
$$

is the same as in (29). Hence, by Theorem A.3 in [3] the system bifurcates from $(u, \alpha)=\left(0, \alpha_{0}\right)$ to a periodic solution; thus the proof is complete.

Remark 3. As an example, let $D_{u}=1, D_{v}=16, \gamma=1$, $\beta=5 / 4$, and $\alpha_{0}=35 / 16$, then from (29), we compute $b=$ $\pi((1141012 / 2205)-(235072 / 3675) \sqrt{5}) \doteq 1176.323160>0$. From Theorem 2, we can conclude that the transition is jump and the system bifurcates to a periodic solution on $\alpha>\alpha_{0}$ which is a repeller (see Figure 1).

3.3. Turing Bifurcation Analysis. In this subsection, we will state the Turing instability for the positive equilibrium $E^{*}$ of model (1). Mathematically speaking, the positive equilibrium $E^{*}$ is Turing instability, which was emphasized by Turing in his pioneering work in 1952 [12]. The Turing bifurcation occurs when

$$
\operatorname{Im}\left(\beta_{k}^{ \pm}\right)=0, \quad \operatorname{Re}\left(\beta_{k}^{ \pm}\right)=0 \quad \text { at } \rho_{k}=\rho_{T} \neq 0
$$

and the wave-number $\sqrt{\rho_{T}}$ satisfies

$$
\rho_{T}=\sqrt{\frac{\operatorname{det}\left(M_{0}\right)}{\mu D_{u}}} .
$$

Hence, Turing instability occurs when the condition either $\operatorname{tr}\left(M_{k}\right)<0$ or $\operatorname{det}\left(M_{k}\right)>0$ is violated.

Since the positive equilibrium $E^{*}$ is stable without diffusion means that $\operatorname{tr}\left(M_{0}\right)<0$ and $\operatorname{det}\left(M_{0}\right)>0$ hold, then $\operatorname{tr}\left(M_{k}\right)<0$ is always true. Therefore, for the emergency of the diffusion-driven instability in model (1), it is needed $\operatorname{det}\left(M_{k}\right)<0$ for some $\rho_{k}>0$. A necessary condition is

$$
a_{11} \mu+d_{22} D_{u}>0
$$

otherwise $\operatorname{det}\left(M_{k}\right)>0$ for all $k>0$ since $\mu D_{u}>0$ and $a_{11} a_{22}-a_{12} a_{21}>0$. And we notice that $\operatorname{det}\left(M_{k}\right)$ achieves its minimum

$$
\min _{k \in \mathbf{R}^{+}} \operatorname{det}\left(M_{k}\right)=a_{11} a_{22}-a_{12} a_{21}-\frac{\left(\mu a_{11}+D_{u} a_{22}\right)^{2}}{4 D_{u} \mu}
$$

at the critical value $\mu_{c}^{2}>0$ where

$$
\mu_{c}^{2}=\frac{\mu a_{11}+D_{u} a_{22}}{2 D_{u} \mu} .
$$

Summarizing the above calculation, we conclude.

Theorem 4. If

$$
\begin{gathered}
a_{11}+a_{22}<0, \\
a_{11} a_{22}-a_{12} a_{21}>0, \\
\mu a_{11}+D_{u} a_{22}>0, \\
\left(\mu a_{11}+D_{u} a_{22}\right)^{2}>4 D_{u} \mu\left(a_{11} a_{22}-a_{12} a_{21}\right),
\end{gathered}
$$

then the positive equilibrium $E^{*}$ of model (1) is Turing unstable.

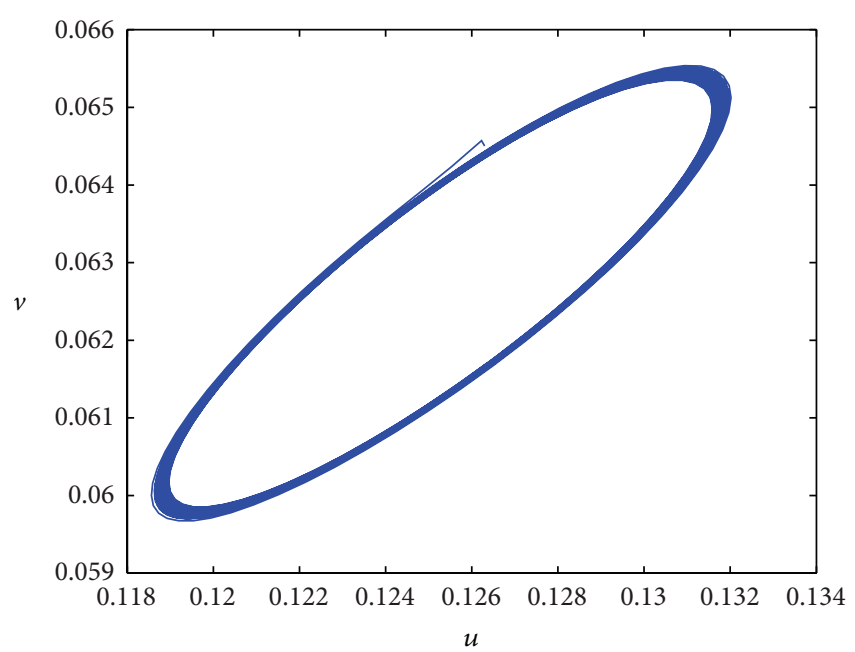

FIGURE 1: The phrase diagram with $D_{u}=1, D_{v}=16, \alpha=35 / 16, \beta=$ $5 / 4$, and $\gamma=1$ illustrating system (4) admits an unstable periodic solution.

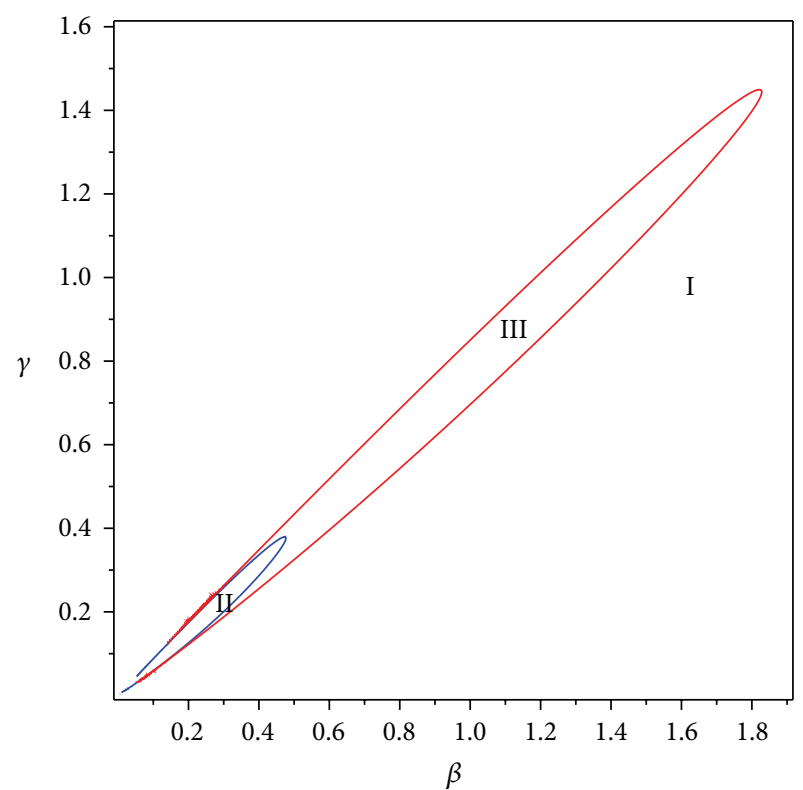

Figure 2: The dispersal relation of $\gamma$ with $\beta$. Parameters: $\alpha=1.8$, $D_{u}=0.02, \sigma=18$. The blue and red curves represent Hopf and Turing bifurcation curves, respectively. They separate the parametric space into three domains, and domain(III) is called Turing space.

In Figure 2, based on the results of Theorem 4, we show the dispersal relation of $\gamma$ with $\alpha$. The blue and red curves represent Hopf and Turing bifurcation curves, respectively. They separate the parametric space into three domains. The outside domain of the Hopf bifurcation curve is stable and the inside domain of the Turing bifurcation curve is unstable. Hence, among these domains, only the domain(III) satisfies the conditions of Theorem 4 and we call domain(III) as Turing space, where the Turing instability occurs and the Turing patterns may be undergone. 


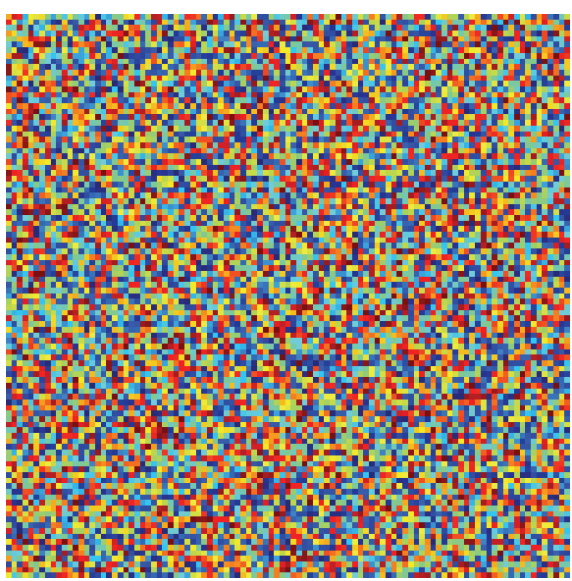

(a)

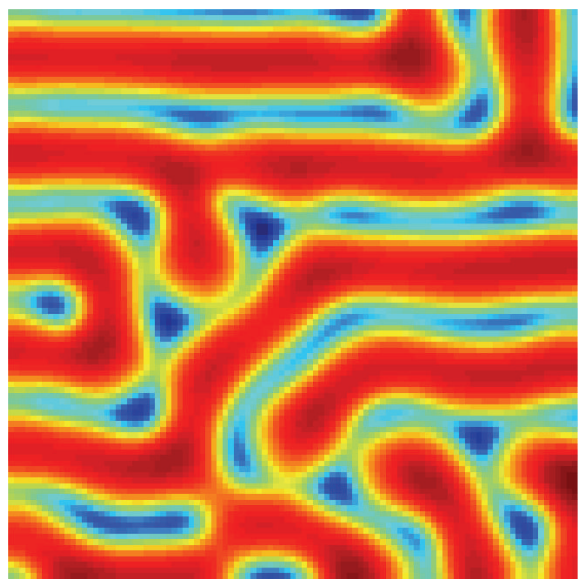

(c)
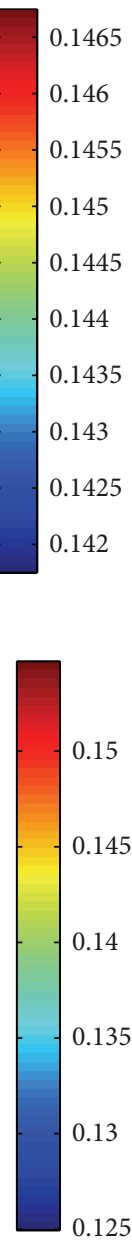

0.125

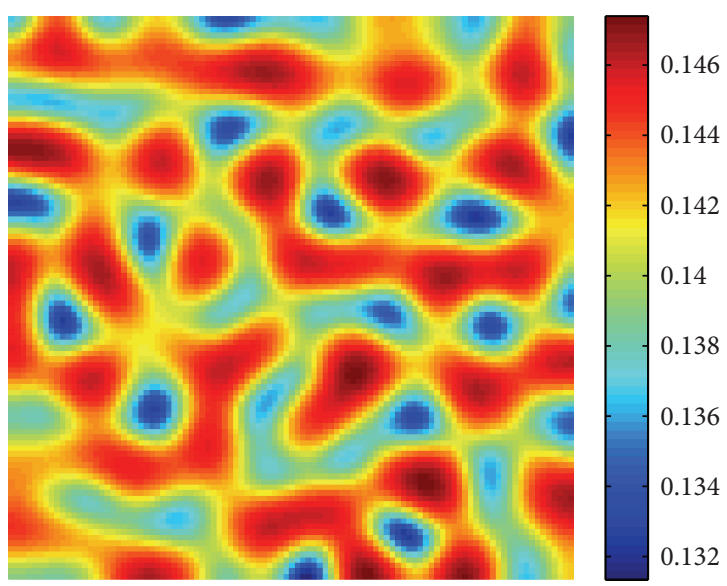

(b)

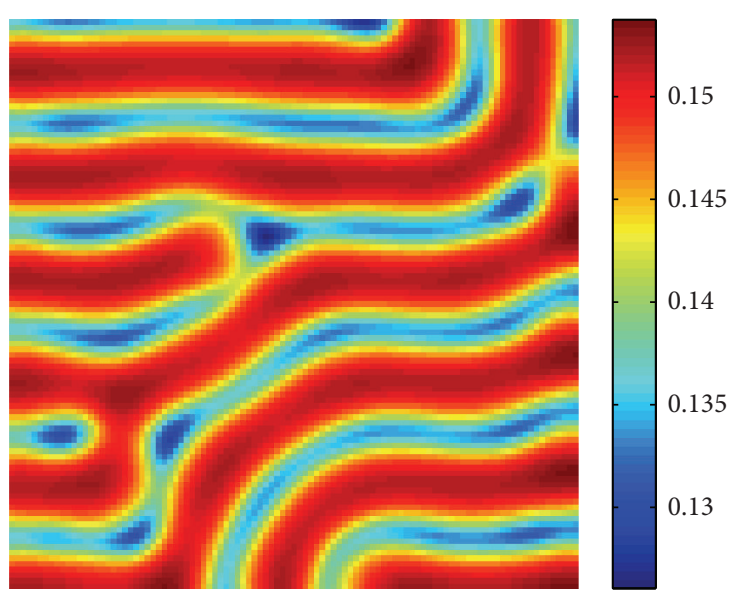

(d)

Figure 3: The process of formation of spiral pattern for $u$ for $(\beta, \gamma)=(0.55,0.44)$; the other parameters are fixed as in (46). Times: (a) $t=0$, (b) $t=100$, (c) $t=500$, and (d) $t=2000$.

\section{Pattern Formation}

In this section, we perform extensive numerical simulations of the spatially extended model (4) in 2-dimensional spaces, and the qualitative results are shown here. Our numerical simulations employ the nonzero initial (5) and the zero-flux boundary conditions (6) with a system size of $L_{x} \times L_{y}$, with $L_{x}=L_{y}=25$ discretized through $x \rightarrow\left(x_{0}, x_{1}, x_{2}, \ldots, x_{n}\right)$ and $y \rightarrow\left(y_{0}, y_{1}, y_{2}, \ldots, y_{n}\right)$, with $n=100$. Other parameters are fixed as

$$
\alpha=1.8, \quad D_{u}=0.02, \quad \sigma=18, \quad h=\frac{1}{4} .
$$

The numerical integration of (4) was performed by fourthorder Runge-Kutta scheme integration [45], with a time step of $\tau=0.01$, and by using the standard five-point approximation for the 2D Laplacian with the zero-flux boundary conditions $[46,47]$. More precisely, the concentrations $\left(u_{i, j}^{n+1}\right)$ at the moment $(n+1) \tau$ at the mesh position $\left(x_{i}, y_{j}\right)$ are given by

$$
\begin{aligned}
u_{i, j}^{(1)}= & u_{i, j}^{n}+\frac{1}{2} \tau F\left(u_{i, j}^{n}\right) \\
u_{i, j}^{(2)}= & u_{i, j}^{n}+\frac{1}{2} \tau F\left(u_{i, j}^{(1)}\right) \\
u_{i, j}^{(3)}= & u_{i, j}^{n}+\tau F\left(u_{i, j}^{(2)}\right) \\
u^{(n+1)}= & \frac{1}{3}\left(-u_{i, j}^{n}+u_{i, j}^{(1)}+2 u_{i, j}^{(2)}+u_{i, j}^{(3)}\right) \\
& +\frac{1}{6} \tau F\left(u_{i, j}^{(3)}\right),
\end{aligned}
$$

where $F(u)$ is defined in (17).

Initially, the entire system is placed in the steady state $\left(u^{*}, v^{*}\right)$, and the propagation velocity of the initial perturbation is thus on the order of $5 \times 10^{-4}$ space units per time unit. And the system is then integrated for 200000 time steps, 


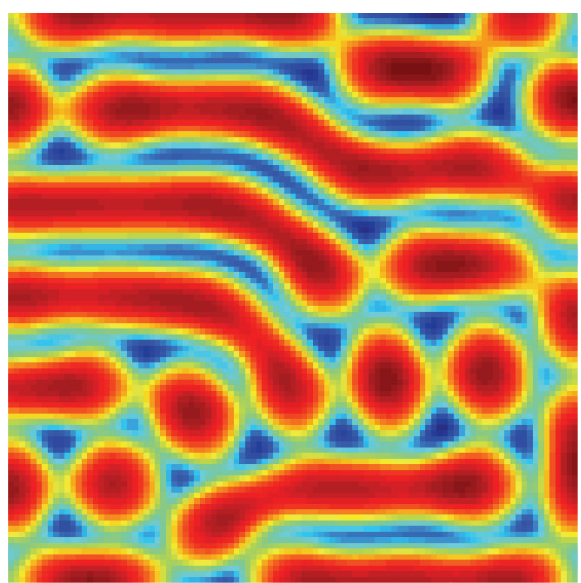

(a)

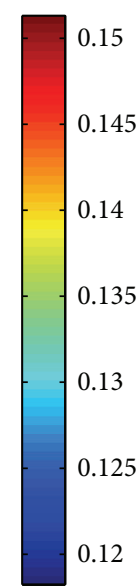

tripes patt

FIGURE 4: Spots-stripes and holes-stripes patter
iterations. Other parameters are fixed as (46).

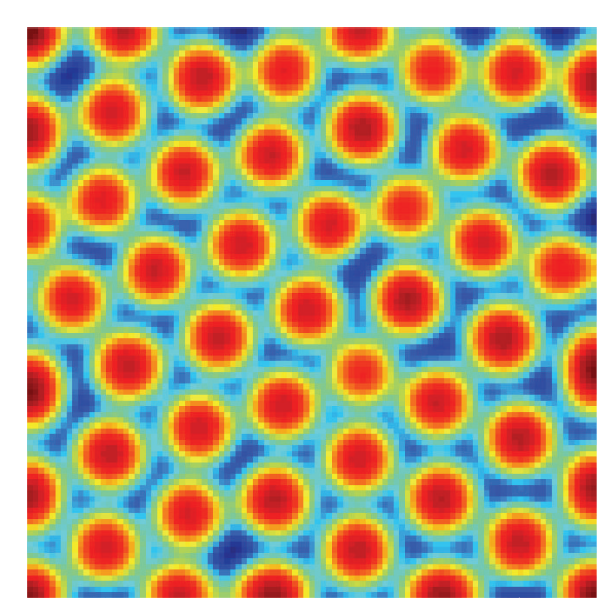

(a)

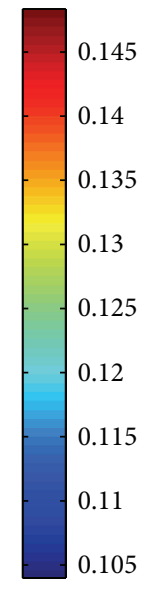

0.105

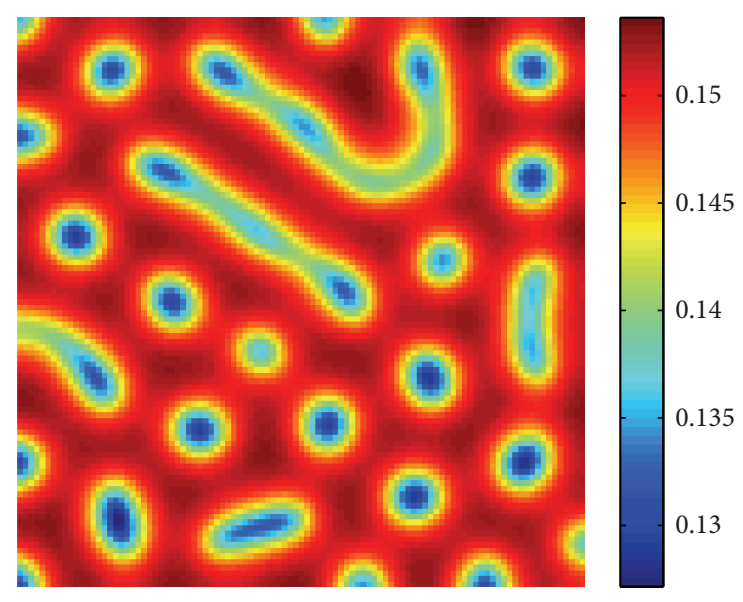

(b)

FIGURE 5: Spots and holes patterns obtained with model (4) for $(\mathrm{a})(\beta, \gamma)=(0.55,0.4)$ and (b) $(\beta, \gamma)=(0.79,0.67)$ at 200000 iterations. Other parameters are fixed as (46).

and the last images are saved. After the initial period during which the perturbation spreads, either the system goes into a time-dependent state or to an essentially steady state (time independent).

In the numerical simulations, different types of dynamics are observed and it is found that the distributions of predator and prey are always of the same type. Consequently, we can restrict our analysis of pattern formation to one distribution. In this section, we show the distribution of prey $u$, for instance. And the parameters are located in the Turing space (cf., Figure 2), the region where Turing instability occurs. We have taken some snapshots with red (blue) corresponding to the high (low) value of prey $u$.

Figure 3 shows the process of pattern formation for model (4) with $\beta=0.55$ and $\gamma=0.44$. In this case, the pattern takes a long time to settle down, starting with a homogeneous state $\left(u^{*}, v^{*}\right)=(0.2800,0.1400)$ (cf., Figure 3(a)), and the random perturbation leads to the formation of stripes and

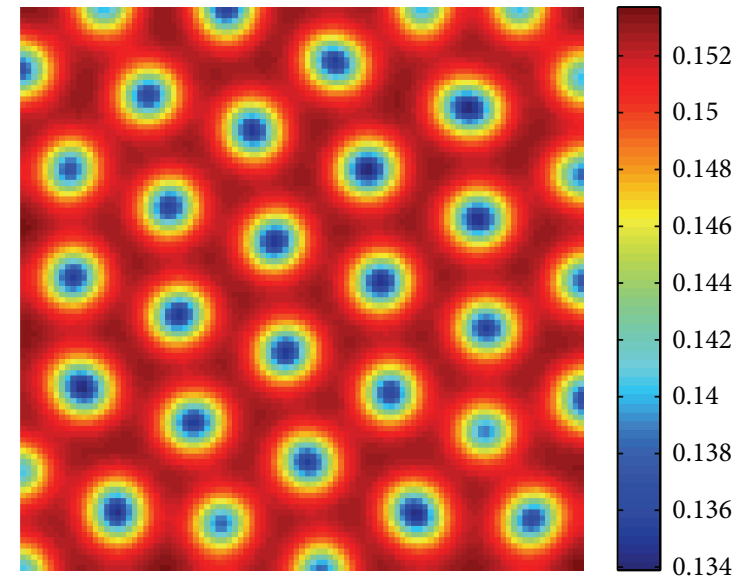

(b) spots (cf., Figures 3(b) and 3(c)) and ends with stripes only (cf., Figure 3(d)), which is time independent.

In Figure 4, we show two spots-stripes patterns obtained with model (4) at 100000 iterations; that is, $t=5000$. These two patterns are similar to each other. With $(\beta, \gamma)=$ $(0.60,0.46)$, in this case, the equilibrium is $\left(u^{*}, v^{*}\right)=$ $(0.2386,0.1316)$ and the spots-stripes pattern is relatively high (cf., Figure $4(\mathrm{a}))$, while with $(\beta, \gamma)=(0.60,0.50)$, the equilibrium is $\left(u^{*}, v^{*}\right)=(0.3291,0.1472)$, at low prey densities (c.f., Figure 4(b)).

In Figure 5, we show the interesting and similartimeindependent patterns which obtained by model (4) at 200 000 iterations. They consist of blue/red spots on a red/blue background. We refer to them as spots (cf., Figure 5(a)) and holes (cf., Figure 5(b)), respectively. In Figure 5(a), with $(\beta, \gamma)=(0.55,0.40),\left(u^{*}, v^{*}\right)=(0.1983,0.1214)$, the hot spots are isolated zones with high prey densities. In this case, the predators are in low density obviously. While with 
$(\beta, \gamma)=(0.79,0.67),\left(u^{*}, v^{*}\right)=(0.3539,0.1497)$, holes are isolated zones with low prey density (Figure 5(b)). In this case, the predators are in high density. From Figure 5(b), one can see that the predators apparently almost occupy the whole spatial domain.

\section{Concluding and Remarks}

In this paper, pattern formation of a spatial model for the growth of bacterial colonies with the two-dimensional space is investigated. Based on both mathematical analysis and numerical simulations, we have found that its spatial pattern includes periodic solutions from Hopf bifurcation and the spotted and striped patterns from Turing bifurcation.

It should be noticed that, if considered in a somewhat broader ecological perspective, our results have an intuitively clear meaning; there has been a growing understanding in the past regarding the dynamics of the system's parameter. From this standpoint, it seems interesting to know that the dynamics vary when the parameter moves across the diagram. From our analysis, the parameters $\gamma$ and $\beta$ play an important role in pattern formation. Our results show that the pattern formation formed by the bacterial colonies model represents rich spatial dynamics which will be useful for studying the dynamic complexity of bacterial ecosystems.

\section{Conflict of Interests}

The authors declare that there is no conflict of interests regarding the publication of this paper.

\section{Acknowledgements}

This research was supported by the Natural Science Foundation of China (61373005 and 11371386), the Doctoral Program of Higher Education of China (20115134110001), the Scientific Research Foundation of CQUT(2012ZD37), and the Fund Project of Zhejiang Provincial Education Department (Y201223449).

\section{References}

[1] E. O. Budrene and H. C. Berg, "Complex patterns formed by motile cells of Escherichia coli," Nature, vol. 349, no. 6310, pp. 630-633, 1991.

[2] E. O. Budrene and H. C. Berg, "Dynamics of formation of symmetrical patterns by chemotactic bacteria," Nature, vol. 376, no. 6535, pp. 49-53, 1995.

[3] M. Badoual, P. Derbez, M. Aubert, and B. Grammaticos, "Simulating the migration and growth patterns of Bacillus subtilis," Physica A, vol. 388, no. 4, pp. 549-559, 2009.

[4] L. Braverman and E. Braverman, "Stability analysis and bifurcations in a diffusive predator-prey system," Discrete and Continuous Dynamical Systems Supplements, pp. 92-100, 2009.

[5] H. Fujikawa and M. Matsushita, "Fractal growth of Bacillus subtilis on agar plates," Journal of the Physical Society of Japan, vol. 58 , no. 11, pp. 3875-3878, 1989.

[6] D. Hartmann, "Pattern formation in cultures of Bacillus subtilis," Journal of Biological Systems, vol. 12, no. 2, pp. 179-199, 2004.
[7] K. Kawasaki, A. Mochizuki, M. Matsushita, T. Umeda, and N. Shigesada, "Modeling spatio-temporal patterns generated by Bacillus subtilis," Journal of Theoretical Biology, vol. 188, no. 2, pp. 177-185, 1997.

[8] E. F. Keller and L. A. Segel, "Initiation of slime mold aggregation viewed as an instability," Journal of Theoretical Biology, vol. 26, no. 3, pp. 399-415, 1970.

[9] A. M. Lacasta, I. R. Cantalapiedra, C. E. Auguet, A. Peñaranda, and L. Ramírez-Piscina, "Modeling of spatiotemporal patterns in bacterial colonies," Physical Review E, vol. 59, no. 6, pp. 70367041, 1999.

[10] M. Mimura, H. Sakaguchi, and M. Matsushita, "Reactiondiffusion modelling of bacterial colony patterns," Physica A, vol. 282, no. 1, pp. 283-303, 2000.

[11] M. Ohgiwari, M. Matsushita, and T. Matsuyama, "Morphological changes in growth phenomena of bacterial colony patterns," Journal of the Physical Society of Japan, vol. 61, no. 3, pp. 816-822, 1992.

[12] A. M. Turing, "The chemical basis of morphogenesis," Philosophical Transactions of the Royal Society of London B, vol. 237, no. 641, pp. 37-72, 1952.

[13] R. Tyson, S. R. Lubkin, and J. D. Murray, "A minimal mechanism for bacterial pattern formation," Proceedings of the Royal Society B, vol. 266, no. 1416, pp. 299-304, 1999.

[14] J. Wakita, K. Komatsu, A. Nakahara, T. Matsuyama, and M. Matsushita, "Experimental investigation on the validity of population dynamics approach to bacterial colony formation," Journal of the Physical Society of Japan, vol. 63, no. 3, pp. 12051211, 1994.

[15] B. Chen and M. X. Wang, "Qualitative analysis for a diffusive predator-prey model," Computers \& Mathematics with Applications, vol. 55, no. 3, pp. 339-355, 2008.

[16] E. N. Dancer and Y. H. Du, "Effects of certain degeneracies in the predator-prey model," SIAM Journal on Mathematical Analysis, vol. 34, no. 2, pp. 292-314, 2002.

[17] Y. H. Du and S. B. Hsu, "A diffusive predator-prey model in heterogeneous environment," Journal of Differential Equations, vol. 203, no. 2, pp. 331-364, 2004.

[18] Y. N. Zhu, Y. L. Cai, S. L. Yan, and W. M. Wang, "Dynamical analysis of a delayed reaction-diffusion predator-prey system," Abstract and Applied Analysis, vol. 2012, Article ID 323186, 23 pages, 2012.

[19] S. L. Yan, X. Z. Lian, W. M. Wang, and Y. B. Wang, "Bifurcation analysis in a delayed diffusive Leslie-Gower model," Discrete Dynamics in Nature and Society, vol. 2013, Article ID 170501, 11 pages, 2013.

[20] X. Q. Zhao, Dynamical Systems in Population Biology, Springer, New York, NY, USA, 2003.

[21] G. Nicolis and I. Prigogine, Self-Organization in Nonequibibrium System, John Wiley \& Sons, New York, NY, USA, 1997.

[22] J. D. Murray, "Discussion: turing's theory of morphogenesis-its influence on modelling biological pattern and form," Bulletin of Mathematical Biology, vol. 52, no. 1-2, pp. 119-152, 1990.

[23] H. Malchow and S. V. Petrovskii, "Dynamical stabilization of an unstable equilibrium in chemical and biological systems," Mathematical and Computer Modelling, vol. 36, no. 3, pp. 307319, 2002.

[24] S. L. Yan, X. Z. Lian, W. M. Wang, and R. K. Upadhyay, "Spatiotemporal dynamics in a delayed diffusive predator model," Applied Mathematics and Computation, vol. 224, pp. 524-534, 2013. 
[25] X. N. Guan, W. M. Wang, and Y. L. Cai, "Spatiotemporal dynamics of a Leslie-Gower predator-prey model incorporating a prey refuge," Nonlinear Analysis: Real World Applications, vol. 12, no. 4, pp. 2385-2395, 2011.

[26] X. Z. Lian, H. L. Wang, and W. M. Wang, "Delay-driven pattern formation in a reaction-diffusion predatorprey model incorporating a prey refuge," Journal of Statistical Mechanics: Theory and Experiment, vol. 2013, no. 4, Article ID P04006, 2013.

[27] W. M. Wang, Y. Z. Lin, L. Zhang, F. Rao, and Y. J. Tan, "Complex patterns in a predator-prey model with self and cross-diffusion," Communications in Nonlinear Science and Numerical Simulation, vol. 16, no. 4, pp. 2006-2015, 2011.

[28] W. M. Wang, W. J. Wang, Y. Z. Lin, and Y. J. Tan, "Pattern selection in a predation model with self and cross diffusion," Chinese Physics B, vol. 20, no. 3, Article ID 034702, 2011.

[29] X. Z. Lian, Y. H. Yue, and H. L. Wang, "Pattern formation in a cross-diffusive ratio-dependent predator-prey model," Discrete Dynamics in Nature and Society, vol. 2012, Article ID 814069, 13 pages, 2012.

[30] R. K. Upadhyay, W. M. Wang, and N. K. Thakur, "Spatiotemporal dynamics in a spatial plankton system," Mathematical Modelling of Natural Phenomena, vol. 5, no. 5, pp. 102-122, 2010.

[31] W. M. Wang, Z. G. Guo, R. K. Upadhyay, and Y. Z. Lin, "Pattern formation in a cross-diffusive Holling-Tanner model," Discrete Dynamics in Nature and Society, vol. 2012, Article ID 828219, 12 pages, 2012.

[32] Y. L. Cai, W. B. Liu, Y. B. Wang, and W. M. Wang, "Complex dynamics of a diffusive epidemic model with strong Allee effect," Nonlinear Analysis: Real World Applications, vol. 14, no. 4, pp. 1907-1920, 2013.

[33] S. Wang, W. B. Liu, Z. G. Guo, and W. M. Wang, "Traveling wave solutions in a reaction-diffusion epidemic model," Abstract and Applied Analysis, vol. 2013, Article ID 216913, 13 pages, 2013.

[34] W. M. Wang, Y. L. Cai, Y. N. Zhu, and Z. G. Guo, "Alleeeffect-induced instability in a reaction-diffusion predator-prey model," Abstract and Applied Analysis, vol. 2013, Article ID 487810, 10 pages, 2013.

[35] Y. Yuan, H. L. Wang, and W. M. Wang, "The existence of positive nonconstant steady states in a reaction: diffusion epidemic model," Abstract and Applied Analysis, vol. 2013, Article ID 921401, 7 pages, 2013.

[36] W. M. Wang, Y. N. Zhu, Y. L. Cai, and W. J. Wang, "Dynamical complexity induced by Allee effect in a predator-prey model," Nonlinear Analysis: Real World Applications, vol. 16, pp. 103-119, 2014.

[37] Y. L. Cai, C. D. Zhao, and W. M. Wang, "Spatiotemporal complexity of a leslie-gower predator-prey model with the weak allee effect," Journal of Applied Mathematics, vol. 2013, Article ID 535746, 16 pages, 2013.

[38] W. M. Wang, Y. L. Cai, M. J. Wu, K. F. Wang, and Z. Q. Li, "Complex dynamics of a reaction-diffusion epidemic model," Nonlinear Analysis: Real World Applications, vol. 13, no. 5, pp. 2240-2258, 2012.

[39] T. Ma and S. H. Wang, Bifurcation Theory and Applications, World Scientific, Singapore, 2005.

[40] T. Ma and S. H. Wang, Stability and Bifurcation of Nonlinear Evolution Equations, 2007.

[41] T. Ma and S. Wang, "Dynamic phase transition theory in PVT systems," Indiana University Mathematics Journal, vol. 57, no. 6, pp. 2861-2889, 2008.
[42] T. Ma and S. H. Wang, "Cahn-Hilliard equations and phase transition dynamics for binary systems," Discrete and Continuous Dynamical Systems B, vol. 11, no. 3, pp. 741-784, 2009.

[43] T. Ma and S. H. Wang, "Phase transitions for the Brusselator model," Journal of Mathematical Physics, vol. 52, no. 3, Article ID 033501, 23 pages, 2011.

[44] C. H. Hsia, T. Ma, and S. Wang, "Rotating Boussinesq equations: dynamic stability and transitions," Discrete and Continuous Dynamical Systems A, vol. 28, no. 1, pp. 99-130, 2010.

[45] G. S. Jiang and C. W. Shu, "Efficient implementation of weighted ENO schemes," Journal of Computational Physics, vol. 126, no. 1, pp. 202-228, 1996.

[46] M. R. Garvie, "Finite-difference schemes for reaction-diffusion equations modeling predator-prey interactions in MATLAB," Bulletin of Mathematical Biology, vol. 69, no. 3, pp. 931-956, 2007.

[47] A. Munteanu and R. V. Solé, "Pattern formation in noisy self-replicating spots," International Journal of Bifurcation and Chaos, vol. 16, no. 12, pp. 3679-3685, 2006. 


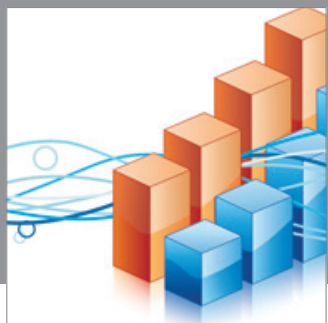

Advances in

Operations Research

mansans

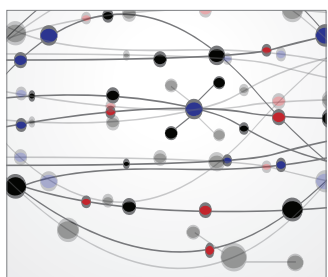

The Scientific World Journal
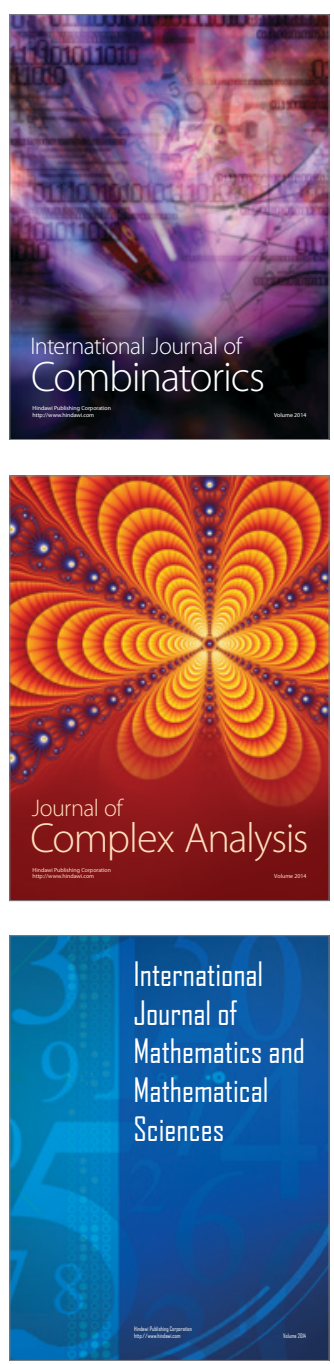
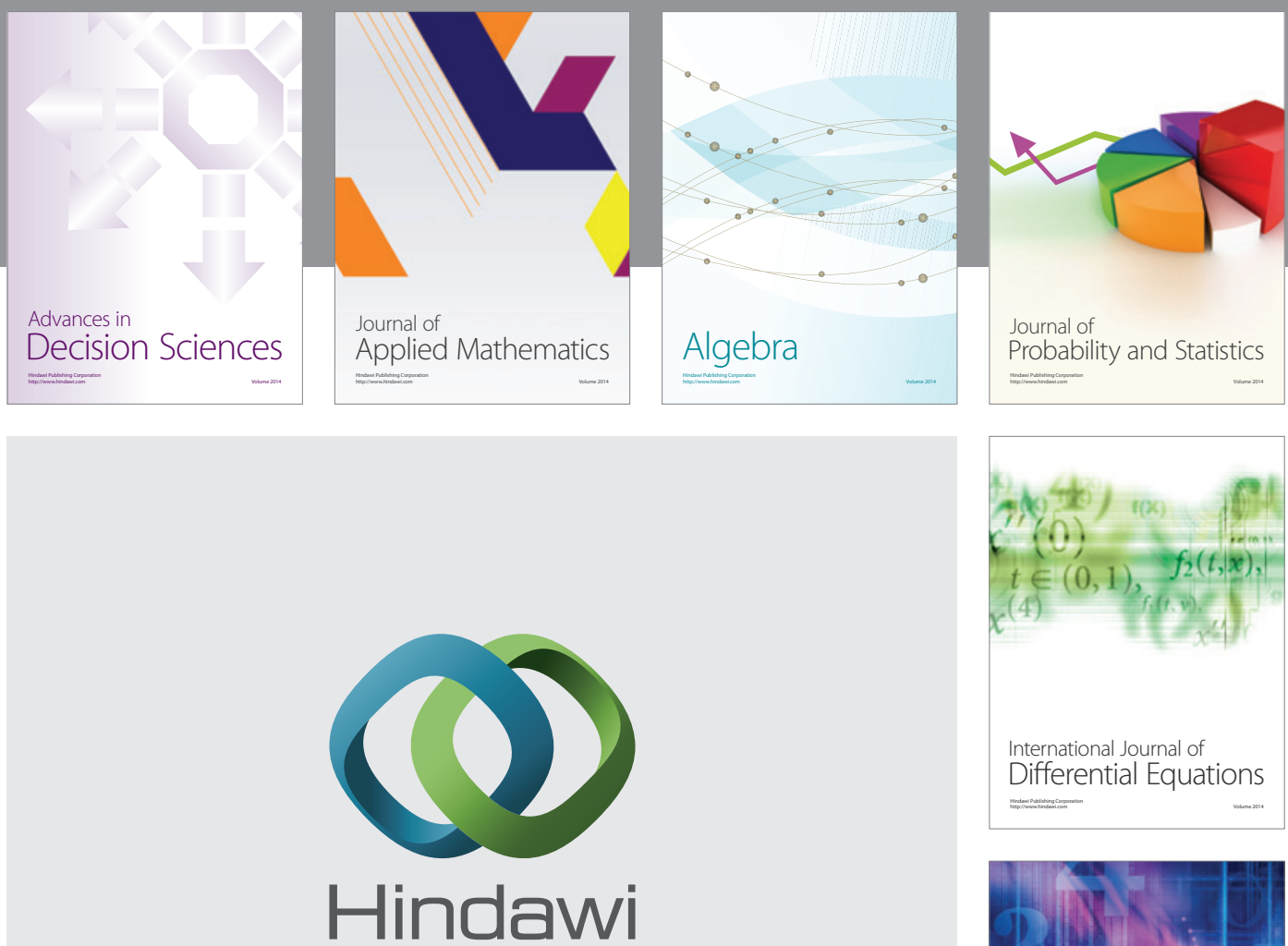

Submit your manuscripts at http://www.hindawi.com
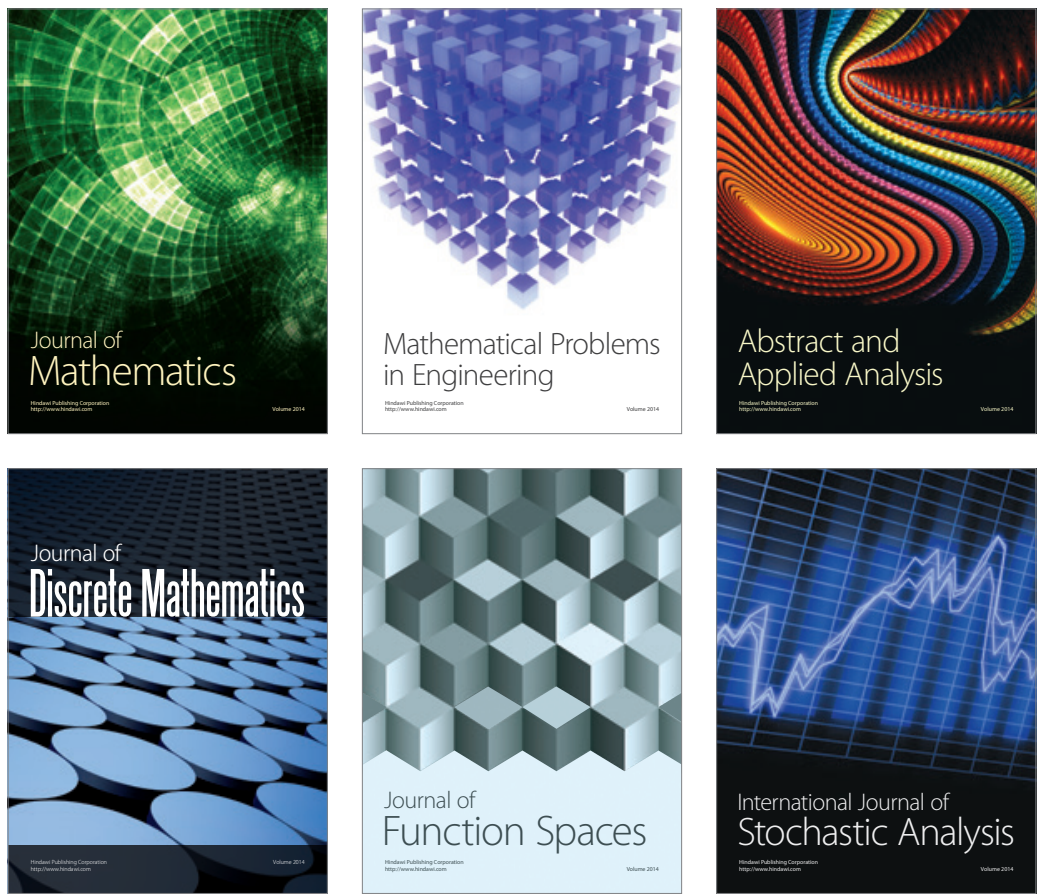

Journal of

Function Spaces

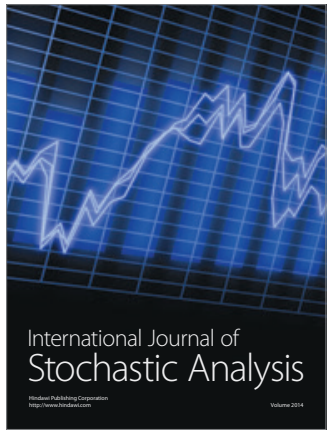

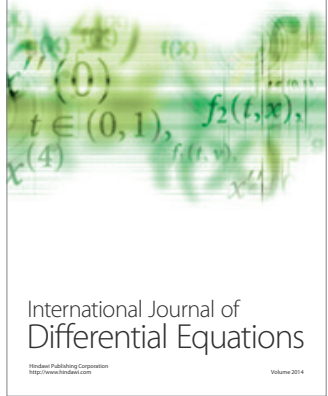
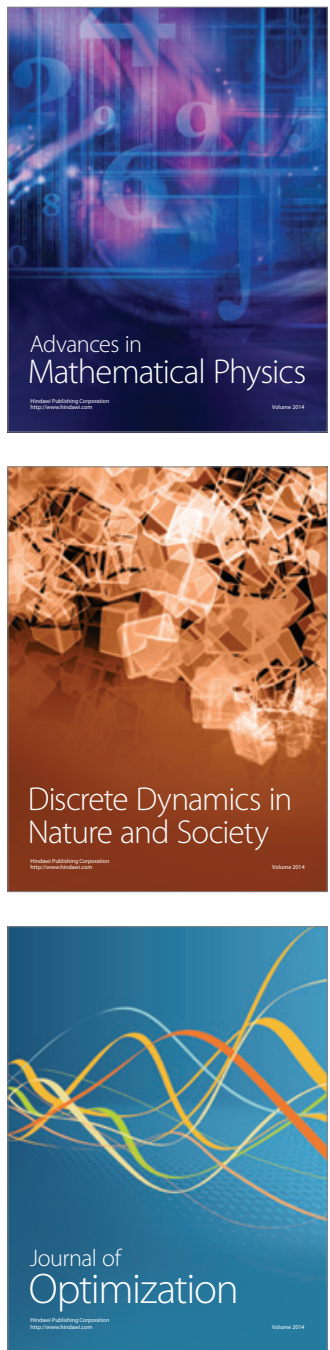